\title{
EMR Data on Mn(III; $S=2$ ) Ions in MnTPPCl Complex Modelled by Microscopic Spin Hamiltonian Approach
}

\author{
K. TADYSZAK ${ }^{a, b, *}$ AND C. RUDOWICZ ${ }^{c, \dagger}$ \\ ${ }^{a}$ NanoBioMedical Centre, Adam Mickiewicz University, Umultowska 85, 61-614 Poznań, Poland \\ ${ }^{b}$ Institute of Molecular Physics, Polish Academy of Sciences, M. Smoluchowskiego 17, 60-179 Poznań, Poland \\ ${ }^{c}$ Visiting Professor: Faculty of Chemistry, Adam Mickiewicz University, Umultowska 89B, 61-614 Poznań, Poland
}

\begin{abstract}
The electron magnetic resonance data on high-spin $(S=2)$ manganese(III) $3 d^{4}$ ion in tetraphenylporphyrinato chloride complex (MnTPPCl) obtained by high-frequency techniques are reanalysed. Preliminary results of semiempirical modeling of the spin Hamiltonian parameters for $\mathrm{Mn}(\mathrm{III})$ in $\mathrm{MnTPPCl}$ are presented. The microscopic spin Hamiltonian approach is utilized to predict the zero-field splitting and the Zeeman electronic parameters. It is found that for $\mathrm{Mn}(\mathrm{III})$ ions in $\mathrm{MnTPPCl}$ matching the experimental spin Hamiltonian parameters and the theoretical ones based on the ligand-field energy levels $\left(\Delta_{i}\right)$ within the ${ }^{5} D$ multiplet only may not be suitable for this system. Contributions due to the levels arising from the higher-lying ${ }^{3} \mathrm{H}$ multiplet need to be taken into account in order to determine the reasonable values of microscopic parameters describing $\mathrm{Mn}$ (III) ions in $\mathrm{MnTPPCl}$.
\end{abstract}

DOI: 10.12693/APhysPolA.132.15

PACS/topics: 33.35.+r, 71.70.Ch, 71.70.Ej, 76.20.+q, 76.30.-v, 76.30.Fc, 87.80.Lg

\section{Introduction}

The transition metal complexes play an important role in chemistry and biology due to their electronic structure. Since the incompletely filled $3 d$ shell leads to paramagnetism, the best method to investigate their magnetic and spectroscopic properties is electron magnetic resonance (EMR, which includes also EPR/ESR) [1-3] based on the effective spin Hamiltonian (SH) $[4,5]$. Due to the large zero-field splitting (ZFS) characteristic for integer spin $3 d^{4}$ and $3 d^{6}$ systems, see e.g. [6, 7], the high-field EMR and high-frequency (HMF-EMR) techniques are best suited for detection of the allowed spin transitions [8-10]. Early studies of SH parameters of $\mathrm{Mn}$ (III) ions in porphyrinic and related complexes have been reviewed in [11], whereas more recent studies include, e.g. [12-14].

Using HMF-EMR Krzystek et al. [11] have obtained complete powder spectra of the high-spin $(S=2)$ manganese(III) $3 d^{4}$ ion in 5,10,15,20-tetraphenyl-21H,23Hporphine chloride complex $(\mathrm{MnTPPCl})$, which exhibits axial symmetry, and elucidated its electronic structure. The axial ZFS parameter (ZFSP) was determined as $D=b_{2}^{0}=-2.31 \mathrm{~cm}^{-1}$ and the $g$-factor as close to 2.00 [11]. Attempts to rationalize these results in terms of the microscopic spin Hamiltonians (MSH) approach have also been made, however, the contributions due to the electronic spin-spin (SS) coupling (SSC) and the fourthrank ZFSPs, admissible for spin $S=2$, have not been taken into account therein [11].

\section{*corresponding author; e-mail: krztad@amu.edu.pl}

${ }^{\dagger}$ On leave of absence from: Institute of Physics, West Pomeranian University of Technology, al. Piastów 17, 70-310 Szczecin, Poland
In this paper we present preliminary results of semiempirical modeling of the $\mathrm{SH}$ parameters for $\mathrm{Mn}$ (III) ions in tetraphenylporphyrinato chloride complexes utilizing the MSH approach developed up to fourth-order perturbation theory $[6,15]$ for $3 d^{4}$ and $3 d^{6}$ systems with $S=2$ within the ground ${ }^{5} \mathrm{D}$ multiplet. Calculations of the $\mathrm{ZFSP} b_{2}^{0}(=D)$ and the Zeeman electronic (Ze) factors $g_{i}$ are carried out for wide ranges of values of the microscopic parameters using the MSH/VBA package [6]. The aim of this study is to elucidate the intrinsic magnetic nature of $\mathrm{Mn}(\mathrm{III})$ ions at tetragonal symmetry sites and verify suitability of the studied crystals for application as high-pressure probes for HMF-EMR [8-10] studies.

\section{Theoretical background}

The theoretical background for this study is provided by the physical Hamiltonians, including the crystal (ligand) field (CF) terms [16-19], and the effective spin Hamiltonians (SH), including the ZFS and Ze terms [15]. The underlying theory has been outlined in the full paper together with pertinent references [20]. Here we only recap the key points and defined the notations used. The physical zero-field splitting (ZFS) within the states of an orbital singlet ground state $\left|\Gamma_{0}\right\rangle\left|S=2, M_{s}\right\rangle$ is due to the combined action of the spin-orbit coupling (SOC) and the electronic spin-spin (SS) coupling acting within the CF states. In the MSH approach only the states arising from the ground ${ }^{5} D(S=2)$ multiplet are considered $[6,7,15]$. Using the complete matrix diagonalization incorporated into the crystal field analysis (CFA) package [21] and its extension CFA/MSH package [22] all states within the whole $3 d^{4}$ and $3 d^{6}$ configuration are taken into account. The role of the contributions to the ZFS parameters (ZFSP) for $\mathrm{Fe}^{2+}$ in several systems arising from the higher lying spin-triplet ${ }^{3} L(S=1)$ states was studied in $[23,24]$. It appears that these contributions may be important, as compared with the ${ }^{5} D$ approximation. Krzystek et al. [11] used a mixed approach, 
i.e. in addition to the second-order perturbation theory terms within the ${ }^{5} D$ approximation, the ZFSP contributions due to one state of the higher spin-triplet ${ }^{3} H$ was taken into account. A question of compatibility of the respective results is considered in this study.

The general form of the Ze and ZFS terms in $\mathrm{SH}$ expressed in terms of the extended Stevens operators (ESO), $O_{k}^{q}$, defined in $[25,26]$ is written as

$$
\begin{aligned}
\tilde{H} & =\tilde{H}_{Z e}+\tilde{H}_{Z F S}=\mu_{\mathrm{B}} \boldsymbol{B} \cdot \boldsymbol{g} \cdot \boldsymbol{S} \\
& +\sum_{k=2,4,6} \sum_{q=-k}^{k} B_{k}^{q} O_{k}^{q}\left(S_{x}, S_{y}, S_{z}\right)=\mu_{\mathrm{B}} \boldsymbol{B} \cdot \boldsymbol{g} \cdot \boldsymbol{S} \\
& +\sum_{k=2,4,6} \sum_{q=-k}^{k} f_{k} b_{k}^{q} O_{k}^{q}\left(S_{x}, S_{y}, S_{z}\right) .
\end{aligned}
$$

Equation (1) is suitable for transition ions at triclinic symmetry sites, whereas for tetragonal symmetry the second-rank ZFS terms in the conventional notation $[4,5]$ used in [11] are

$$
H_{Z F S}=B_{2}^{0} O_{2}^{0}=\frac{b_{2}^{0} O_{2}^{0}}{3}=D\left[S_{z}^{2}-\frac{S(S+1)}{3}\right] .
$$

The conversion relation applies $D=3 B_{2}^{0}=b_{2}^{0}$. In the case of the negative axial ZFSP $b_{2}^{0}<0$, which will be discussed in here, the ground effective spin state is $|2, \pm 2\rangle$, whereas for $b_{2}^{0}>0$ the state $|2,0\rangle$. The modelling of the ZFSPs and the $g$-factors, measured by HMF-EMR [11], are carried out for $\mathrm{Mn}(\mathrm{III})$ ions in MnTPPCl using the $\mathrm{MSH} / \mathrm{VBA}$ package [6].

\section{Electronic structure of $\mathrm{Mn}$ (III) ions in $\mathrm{MnTPPCl}$}

The available crystallographic, magnetic, and spectroscopic data on Mn(III) ions in MnTPPCl have been summarized in [20]. Mn(III) ions occupy the distorted octahedral sites with tetragonal $C_{4 v}$ symmetry. The expected energy levels and the respective wave functions in Cartesian coordinates expected for $\mathrm{Mn}$ (III) ions at octahedral and tetragonal sites [27] are depicted in Fig. 1. The energy relations in terms of the conventional $[17,19] \mathrm{CF}$ parameters (CFPs) provided in [11] are also indicated. Assignment of the wave functions corresponding to the energy levels in Fig. 1 is adapted from [11] as follows: ${ }^{5} B_{1 g}=d_{x^{2}-y^{2}},{ }^{5} A_{1 g}=d_{z}^{2},{ }^{5} E_{g}=\left(d_{x z}, d_{y z}\right),{ }^{5} B_{2 g}=d_{x y}$, whereas the energy spacing were originally [11] denoted as: $\delta_{1}={ }^{5} E_{g} \rightarrow{ }^{5} B_{2 g}, \delta_{2}={ }^{5} B_{1 g} \rightarrow{ }^{5} A_{1 g}, \delta_{3}={ }^{5} B_{1 g} \rightarrow{ }^{3}$ $E$, and $\Delta={ }^{5} B_{1 g} \rightarrow{ }^{5} B_{2 g}$. The equivalence of $\delta_{i}$ and $\Delta$ with the energy spacing $\Delta_{i}$ used in the $\mathrm{MSH} / \mathrm{VBA}$ package [6] is given by: $\Delta_{1}=\Delta_{2} \approx \Delta-\delta_{1}, \Delta_{3} \approx \Delta$, $\Delta_{4} \approx \delta_{2}$.

The sequence of energy levels (ELs) adopted in [11], based on the literature values of the conventional CFPs, and correspondence between the wave functions in Fig. 1 and those in the notation $\left|L, \pm M_{L}\right\rangle$ defined in the $\mathrm{MSH} / \mathrm{VBA}$ package $[6,15]$ is provided in [20]. Guided by the predictions of the ELs [11], an initial set of input (i.e. microscopic) parameters for our calculations is selected as $\left(\right.$ in $\left.\mathrm{cm}^{-1}\right): \Delta_{1}=\Delta_{2}=22500, \Delta_{3}=25000$,

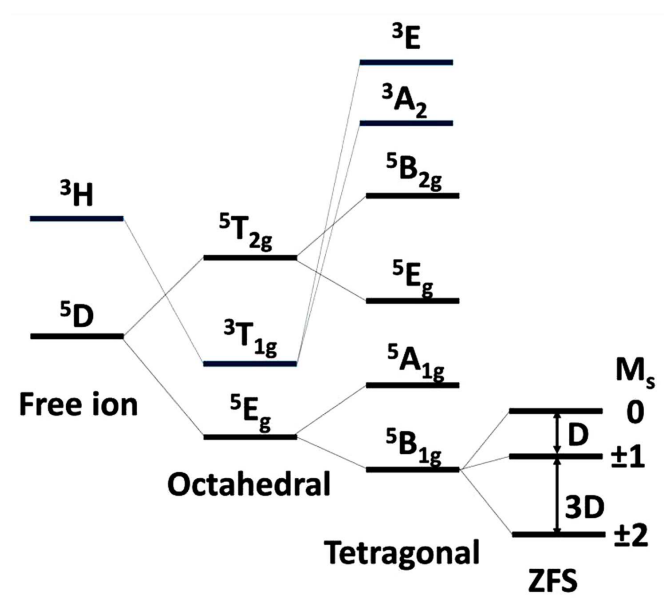

Fig. 1. Schematic energy levels (not in scale) arising from the free ion (FI) ${ }^{5} D\left(d^{4}\right)$ term for $\mathrm{Mn}$ (III) ions at octahedral $(\mathrm{OH})$ and axially elongated tetragonal (TE) complexes exhibiting negative ZFSP $D$. For axially compressed tetragonal complexes positive ZFSP $D$ is expected [27] resulting in inversion of spin levels.

$\Delta_{4}=21500$, the SOC constant $\lambda$ in the range 50 to 110 , and the SSC constant $\rho=0.18$ [28]. Additionally, for tetragonal symmetry the mixing coefficients $[6,15]$ satisfy the relations: $q=1, s=0$. Note that the SOC constant $\lambda$ in crystal is reduced from the free ion value $\lambda_{0}: \lambda=k \times \lambda_{0}, k \leq 1$, due to the orbital (covalency) reduction $[16,17,19]$. The values $\lambda_{0}$ available in literature range from: $83.4 \mathrm{~cm}^{-1}$ [29], $88 \mathrm{~cm}^{-1}[6,30,31]$, $89 \mathrm{~cm}^{-1}$ [32], $90.4 \mathrm{~cm}^{-1}$ [16], to $110 \mathrm{~cm}^{-1}$ [28].

To take into account the contributions to the ZFSPs and $g$-factors arising from the higher-lying ${ }^{3} H$ multiplet states (see Fig. 1) the relation [11]:

$$
D^{\prime}=-\frac{\xi^{2}}{4 \Delta E\left({ }^{3} E-{ }^{5} A_{1}\right)}
$$

for ${ }^{5} B_{1}$ ground state, where $\xi=2 S \lambda=4 \lambda$, is utilized. Note that for the cases of $3 d^{4}$ (e.g. $\mathrm{Cr}^{2+}$ ) and $3 d^{6}$ (e.g. $\left.\mathrm{Fe}^{2+}\right)$ ions, where the spin-triplet states are much higher in energy than the spin-quintet states [33], the ${ }^{5} D$ approximation can be considered sufficiently reliable. However, the study [11] indicates that this condition may not be well satisfied for the case of $\mathrm{Mn}$ (III) ion at tetragonal $C_{4 v}$ symmetry sites in MnTPPCl.

\section{MSH modelling of the ZFS parameters for $\mathrm{Mn}(\mathrm{III})$ ions in MnTPPCl}

The energy levels for $\mathrm{Mn}(\mathrm{III})$ ion at tetragonal symmetry sites in MnTPPCl (see Fig. 1) correspond to the energy level scheme denoted as "case 2" in the MSH/VBA program [6, 15], which is utilized for MSH modelling of the ZFSPs and the $g$-tensor components for $\mathrm{Mn}$ (III) ions in MnTPPCl.Calculations carried out using the initial set of input parameters (see above) yielded the variation of the total ZFSPs versus $\lambda$ depicted in Fig. 2, whereas the specific contributions of the ZFSPs are listed in Table I. Since the experimental values were determined [11] 
TABLE I

The contributions to the ZFSPs (in $\mathrm{cm}^{-1}$ ) calculated for the input parameter values as listed in text $(\lambda=$ $\left.80 \mathrm{~cm}^{-1}\right)$.

\begin{tabular}{c|c||c|c}
\hline \hline$b_{2}^{0}(\rho)$ & -0.54 & $b_{4}^{0}\left(\lambda^{4}\right)$ & $7.46 \times 10^{-5}$ \\
$b_{2}^{0}\left(\lambda^{2}\right)$ & -0.74 & $b_{4}^{0}\left(\rho^{2}\right)$ & $1.8 \times 10^{-5}$ \\
$b_{2}^{0}\left(\lambda^{3}\right)$ & -0.006 & $b_{4}^{0}\left(\rho \cdot \lambda^{2}\right)$ & $7.79 \times 10^{-5}$ \\
$b_{2}^{0}\left(\lambda^{4}\right)$ & $1.88 \times 10^{-4}$ & total $b_{4}^{0}$ & $1.7 \times 10^{-4}$ \\
\cline { 2 - 3 }$b_{2}^{0}\left(\rho^{2}\right)$ & $1.65 \times 10^{-6}$ & $b_{4}^{4}\left(\lambda^{4}\right)$ & $-3.39 \times 10^{-4}$ \\
$b_{2}^{0}(\rho \cdot \lambda)$ & -0.0058 & $b_{4}^{4}\left(\rho^{2}\right)$ & $-1.36 \times 10^{-4}$ \\
total $b_{2}^{0}$ & -1.29 & $b_{4}^{4}\left(\rho \cdot \lambda^{2}\right)$ & $-6.3 \times 10^{-4}$ \\
& & total $b_{4}^{4}$ & -0.0011
\end{tabular}

TABLE II

The contributions to the $g$-tensor components $g_{\|}$and $g_{\perp}$ (dimensionless) calculated for the input parameter values as listed in text; $g_{e}$ denotes the free-ion value $2.0023(\lambda=$ $\left.80 \mathrm{~cm}^{-1}\right)$.

\begin{tabular}{c|c|c|c|c}
\hline \hline$g_{i}$ & $g_{e}+g_{i}(\lambda)$ & $g_{i}\left(\lambda^{2}\right)$ & $g_{i}\left(g_{e} \lambda^{2}\right)$ & Total \\
\hline$g_{x}=g_{y}=g_{\perp}$ & 1.9952 & $1.3 \times 10^{-5}$ & $-27 \times 10^{-5}$ & 1.995 \\
$g_{z}=g_{\|}$ & 1.9767 & $-5.8 \times 10^{-5}$ & $-27 \times 10^{-5}$ & 1.976
\end{tabular}

as $g_{\|}=1.98(2)$ and $g_{\perp}=2.005(3)$, we have rounded the theoretical values in Table II accordingly. Full analysis will be given in [20].

\section{Discussion}

Analysis of the MSH modelling results in Tables I and II, which illustrate the role of various contributions enabling the following comments. It appears that for
$\mathrm{Mn}$ (III) ions at tetragonal symmetry sites the dominant contributions to $b_{2}^{0}$ arise from $b_{2}^{0}\left(\lambda^{2}\right)$ and $b_{2}^{0}(\rho)$, whereas to $g_{i}$ from $g_{i}(\lambda)$. Unlike for $\mathrm{Fe}(\mathrm{II})$ ions the contributions arising from the higher-order perturbation theory play insignificant role. This is due to the different sequence and magnitude of the energy levels $[6,7]$.

A survey of selected data available for $\mathrm{Mn}$ (III) ions in porphyrinic and related complexes [20] yields the values of $b_{2}^{0}(=D)$ in the range $-1.5 \mathrm{~cm}^{-1}<b_{2}^{0}<-3 \mathrm{~cm}^{-1}$. Hence, Fig. 2 and Table I reveal that agreement with the experimental $b_{2}^{0}(=D)$ values may be achieved for some complexes, however, it is not the case of MnTPPCl [11]. It appears that the ZFSPs estimated based on the ligandfield energy levels $\left(\Delta_{i}\right)$ within the ${ }^{5} D$ multiplet only may not be suitable for this system. To match tentatively the experimental $\mathrm{SH}$ parameters and the theoretical ones for $\mathrm{Mn}$ (III) ions in $\mathrm{MnTPPCl}$ we take into account the specific contribution $D^{\prime}$ [11], which is due to some levels arising from the higher-lying ${ }^{3} H$ multiplet. The values of the total $b_{2}^{0}(=D)$ calculated including the contribution $D^{\prime}$ (see Fig. 2) show that an agreement is achievable for a reasonable values of $\lambda$ (in crystal) around $80 \mathrm{~cm}^{-1}$ or less if the energy difference $\Delta E\left({ }^{3} E-{ }^{5} A_{1}\right)$ would decrease. Detailed results of the semiempirical modeling of the SH parameters aimed at elucidation of the intrinsic magnetic nature of $\mathrm{Mn}(\mathrm{III})$ ions at tetragonal symmetry sites in $\mathrm{MnTPPCl}$ and related systems as well as full analysis will be provided in [20]. The question of suitability of the studied crystals for application as highpressure probes for HMF-EMR [8-10] studies will be also discussed therein.
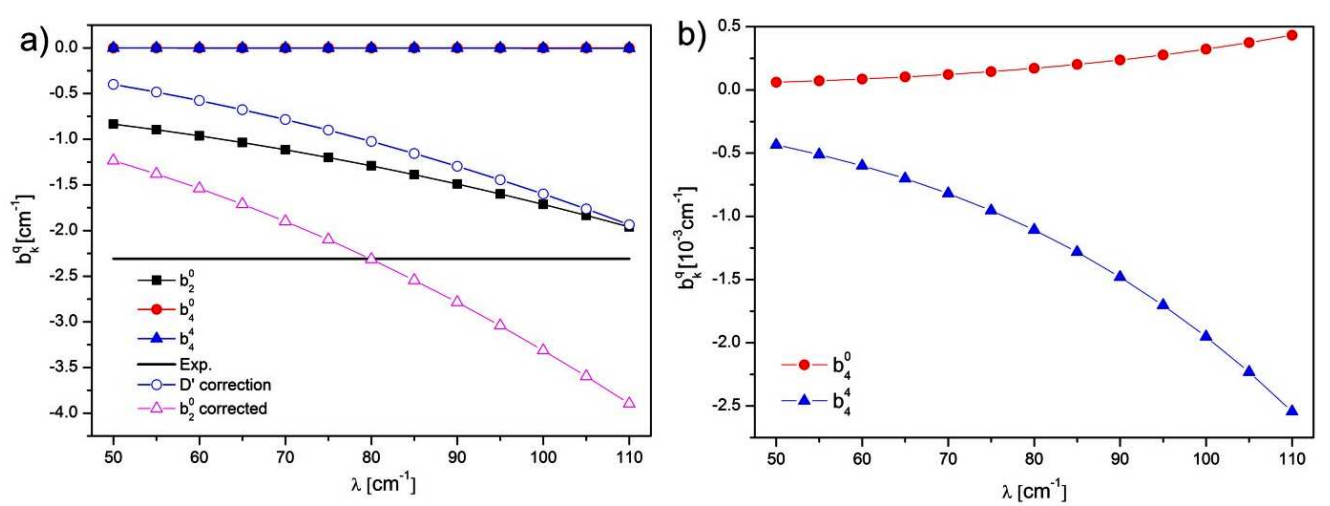

Fig. 2. Variation of the ZFSPs (in $\mathrm{cm}^{-1}$ ) versus $\lambda$ for the input parameters defined in text: (a) all ZFSPs, (b) fourthrank ZFSPs magnified from part (a). Experimental value $b_{2}^{0}(=D) \approx-2.31 \mathrm{~cm}^{-1}$ for $\mathrm{Mn}$ (III) in MnTPPCl [11] is indicated by a straight line. The magenta line (open triangles) represents the values of $b_{2}^{0}(=D)$ calculated including the contribution $D^{\prime}$ (see text).

Here we only mention recent extensive experimental and theoretical studies [34] of peroxomanganese(III) complex supported by a scorpionate ligand, which exhibits orthorhombic site symmetry. The authors [34] used electronic absorption, EMR, low-temperature magnetic circular dichroism (MCD), and variable-temperature variable-field (VTVH) MCD spectroscopies. Theoretical calculations [34] employed several density functional theory (DFT) methods, namely, time dependent (TD-
DFT), coupled-perturbed (CP-DFT) DFT, and multireference $a b$ initio CASSCF/NEVPT2. Detailed discussion of the results [34] will be provided in [20]. Here we mention only that the CP-DFT computations have revealed that the dominant contribution to $D$ was through spin-orbit coupling $\left(D_{S O C}=-1.37 \mathrm{~cm}^{-1}\right)$, whereas the spin-spin coupling contribution was found non-negligible $\left(D_{S S}=-0.32 \mathrm{~cm}^{-1}\right)$. However, the value of $D_{S O C}$ was slightly underestimated relative to the ex- 
perimental $D=-2.0 \mathrm{~cm}^{-1}$. Interestingly, the stateaveraged CASSCF/NEVPT2 method predicted overestimated value: $D_{S O C}=-2.91 \mathrm{~cm}^{-1}$.

Hence, a caution is needed when assessing the DFT results as well as an overall importance of the higher-lying spin-triplet states. Note that other states not included in $D^{\prime}$ may also contribute to the total $D$, whereas various contributions may cancel each other [23, 24, 34]. The study [34] indicates also that the SSC constant $\rho=0.18 \mathrm{~cm}^{-1}$ adopted by us yields proportionally larger values of the ratio $D_{S S} / D_{S O C}=0.73$ than that predicted in [34] for peroxomanganese(III) complex $D_{S S} / D_{S O C}=$ 0.23 . To clarify the role of the SS contributions $b_{2}^{0}(\rho)$ more reliable values of the SSC constant is needed. In view of the site symmetry lower than axial in peroxomanganese(III) complex [34] these preliminary conclusions on applicability of DFT-based methods might not be fully justified. In order to make a more accurate assessment, calculations using the CFA or CFA/MSH package $[21,22]$ within the whole $3 d^{4}$ configuration and incorporating lower symmetry would be indispensable, which are beyond the scope of this paper. Once new theoretical results become available, the present predictions may be verified and fine-tuned, yielding more reliable ranges of the microscopic parameter values feasible for $\mathrm{Mn}$ (III) ions in MnTPPCl and other structurally related systems. This work is in progress.

\section{Acknowledgments}

This work was partially supported by the research grants from the Polish National Science Center: DEC-2012/04/M/ST3/00817 (Cz.R.) and UMO2014/15/B/ST4/ 04946 (K.T.).

\section{References}

[1] J.R. Pilbrow, Transition Ion Electron Paramagnetic Resonance, Clarendon Press, Oxford 1990.

[2] F.E. Mabbs, D. Collison, D. Gatteschi, Electron Paramagnetic Resonance of d Transition Metal Compounds, Elsevier, Amsterdam 1992.

[3] J.A. Weil, J.R. Bolton, J.E. Wertz, Electron Paramagnetic Resonance: Elementary Theory and Applications, Wiley, New York 1994.

[4] C. Rudowicz, S.K. Misra, Appl. Spectrosc. Rev. 36, 11 (2001).

[5] C. Rudowicz, M. Karbowiak, Coord. Chem. Rev. 287, 28 (2015).

[6] C. Rudowicz, H.W.F. Sung, Physica B 337, 204 (2003).

[7] M. Zając, I.E. Lipiński, C. Rudowicz, J. Magn. Magn. Mater. 401, 1068 (2016).

[8] T. Sakurai, K. Fujimoto, R. Goto, S. Okubo, H. Ohta, Y. Uwatoko, J. Magn. Reson. 223, 41 (2012).

[9] J. Telser, J. Krzystek, A. Ozarowski, J. Biol. Inorg. Chem. 19, 297 (2014).
[10] P. Gnutek, C. Rudowicz, H. Ohta, T. Sakurai, Polyhedron 102, 261 (2015).

[11] J. Krzystek, J. Telser, L.A. Pardi, D.P. Goldberg, B.M. Hoffman, L.C. Brunel, Inorg. Chem. 38, 6121 (1999).

[12] R. Takeda, K. Koizumi, M. Shoji, H. Nitta, S. Yamanaka, M. Okumura, K. Yamaguchi, Polyhedron 26, 2309 (2007).

[13] M. Horitani, H. Yashiro, M. Hagiwara, H. Hori, J. Inorg. Biochem. 102, 781 (2008).

[14] S.C. Hunter, A.A. Podlesnyak, Z.-L. Xue, Inorg. Chem. 53, 1955 (2014).

[15] C. Rudowicz, Y.Y. Zhou, Comput. Chem. 21, 45 (1997).

[16] C.A. Morrison, R.P. Leavitt, in: Handbook on the Physics and Chemistry of Rare Earth, Eds. K.A. Gschneidner, Jr., L. Eyring, Vol. 5, NorthHolland, Amsterdam 1982.

[17] B.N. Figgis, M.A. Hitchman, Ligand Field Theory and Its Applications, Wiley-VCH, New York 2000.

[18] J. Mulak, Z. Gajek, The Effective Crystal Field Potential, Elsevier Sci., Oxford 2000.

[19] M. Wildner, M. Andrut, C. Rudowicz, in: Spectroscopic Methods in Mineralogy - EMU Notes Mineralogy, Eds. A. Beran, E. Libowitzky, Eötvös Univ. Press, Budapest 2004, p. 93.

[20] C. Rudowicz, K. Tadyszak, , H. Ohta, T. Sakurai, J. Inorg. Biochem. 175, 36 (2017).

[21] Y.Y. Yeung, C. Rudowicz, Comput. Chem. 16, 207 (1992).

[22] Z.-Y. Yang, Y. Hao, C. Rudowicz, Y.-Y. Yeung, J. Phys. Condens. Matter 16, 3481 (2004).

[23] Y.-Y. Zhou, C.-L. Li, Phys. Rev. B 48, 16489 (1993).

[24] C.-Y. Jiang, M.-L. Du, Y.-Y. Zhou, Phys. Rev. B 50, 949 (1994).

[25] C. Rudowicz, J. Phys. C Solid State Phys. 18, 1415 (1985); Erratum: ibid., 18, 3837 (1985).

[26] C. Rudowicz, C.Y. Chung, J. Phys. Condens. Matter 16, 5825 (2004).

[27] H. Miyasaka, A. Saitoh, S. Abe, Coord. Chem. Rev. 251, 2622 (2007).

[28] C. Rudowicz, B. Konkiewicz, Acta Phys. Pol. A 47, 291 (1975).

[29] J. Sugar, C. Corliss, J. Phys. Chem. Ref. Data 14, Suppl. 2, 664 (1985).

[30] A. Abragam, B. Bleaney, Electron Paramagnetic Resonance of Transition Ions, Clarendon Press, Oxford 1970.

[31] J. Krzystek, G.J. Yeagle, J.-H. Park, R.D. Britt, M.W. Meisel, L.-C. Brunel, J. Telser, Inorg. Chem. 42, 4610 (2003).

[32] R. Boča, Coord. Chem. Rev. 248, 757 (2004).

[33] C. Rudowicz, Y.-Y. Zhou, J. Phys. Chem. Solids 60, 17 (1999).

[34] H.E. Colmer, R.A. Geiger, D.F. Leto, G.B. Wijeratne, V.W. Day, T.A. Jackson, Dalton Trans. 43, 17949 (2014). 